Les sources de l'Histoire des Mines : Nouveaux outils, Nouvelles approches

\title{
La sauvegarde de la mémoire de Charbonnages de France. Une priorité de la Direction des archives de France
}

The preservation of the memory of Charbonnages de France. A major issue for the Direction des archives de France

Pascal Even

\section{OpenEdition}

Journals

Édition électronique

URL : http://journals.openedition.org/dht/808

DOI : $10.4000 /$ dht. 808

ISSN : 1775-4194

Éditeur :

Centre d'histoire des techniques et de l'environnement du Cnam (CDHTE-Cnam), Société des élèves du CDHTE-Cnam

\section{Édition imprimée}

Date de publication : 1 décembre 2008

Pagination : 30-39

ISBN : 978-2-95-30779-2-6

ISSN : 0417-8726

\section{Référence électronique}

Pascal Even, «La sauvegarde de la mémoire de Charbonnages de France. Une priorité de la Direction des archives de France ", Documents pour l'histoire des techniques [En ligne], 16 | $2^{\mathrm{e}}$ semestre 2008, mis en ligne le 06 octobre 2010, consulté le 08 septembre 2020. URL : http://journals.openedition.org/dht/ 808 ; DOI : https://doi.org/10.4000/dht.808 


\title{
La sauvegarde de la mémoire de Charbonnages de France. Une priorité de la Direction des archives de France
}

\author{
Pascal Even \\ Direction des archives de France
}

\begin{abstract}
RÉSUMÉ
La sauvegarde des archives de Charbonnages de France constitue très certainement un exemple réussi d'une politique volontariste menée tant par l'administration des archives que par les responsables de l'entreprise afin d'assurer la conservation pour les générations futures d'un patrimoine écrit particulièrement important pour la connaissance de l'histoire économique et sociale contemporaine. L'histoire de la mine dans notre pays et sa dimension sociale occupent en effet une place privilégiée dans la construction de la mémoire contemporaine et c'est ce qui explique le souci des responsables de Charbonnages de France, confrontés à la disparition programmée de leur entreprise, d'engager avec la Direction des archives de France un programme de tri, d'éliminations et de versements de leurs archives. Cette campagne a permis de diriger, au cours des dernières années, l'essentiel de ce patrimoine vers les services publics d'archives. L'article évoque les différentes solutions adoptées dans les trois bassins hovillers du Nord-Pas-de-Calais, de Lorraine et du Centre-Midi pour assurer la collecte, le traitement et la mise en valeur des archives de la mine mais aussi les partenariats développés avec les acteurs de l'après mine, le Bureau des recherches géologiques et minières, ou les structures associatives qui militent pour la mémoire de la mine.
\end{abstract}

Résumés et mots clés en anglais sont regroupés en fin de volume, accompagnés des mots clés français

D ans l'éventail des sources dont disposent les chercheurs sur l'histoire des mines de charbon, une place toute particulière revient sans conteste au formidable fonds d'archives constitué par les Hovillères depuis leur création. Ensemble considérable qui couvre tous les aspects de l'exploitation de la houille, de l'histoire des bassins et des puits, qu'il s'agisse de la stratégie et du fonctionnement des compagnies minières privées puis de Charbonnages de France et des houillères de bassin après les mesures de nationalisation de 1946, de l'histoire des techniques ou de la vie des mineurs.

Avec la diminution d'activité progressive de l'exploitation du charbon à partir des années 1960 et les premières fermetures de puits, commence à se poser la question de la sauvegarde d'une mémoire emblématique non seulement pour les historiens mais surtout pour les élus et la population des bassins miniers. Or à côté des bâtiments et des installations techniques, des savoirs et des témoignages, les archives partici- pent éminemment à cette mémoire. La décision de suppression définitive de Charbonnages de France et les étapes successives de sa mise en œuvre ont rendu de ce fait indispensable une concertation étroite entre l'entreprise et la Direction des archives de France, coopération renforcée au cours des dernières années au fur et à mesure que se rapprochait la date de disparition de l'entreprise.

D'aucuns regretteront sans doute que ce partenariat ait tardé à se mettre en place et que des pertes documentaires doivent être déplorées ; on évoque régulièrement le sort des archives de certains sites miniers d'Auvergne précipitées dans les puits au moment de leur fermeture. En dépit de difficultés inhérentes à la mise en œuvre de ce plan ambitieux, ce partenariat développé à tous les niveaux du réseau des archives de France, s'est traduit par le versement en quelques années d'un volume particulièrement important d'archives dans les dépôts publics. À quelques mois de la disparition effective de Charbonnages de France, la 
présente journée d'étude offre, dans ces conditions, une opportunité pour dresser, sinon un bilan complet, du moins un premier bilan de la campagne de sauvegarde engagée au cours des dernières années par la Direction des archives de France et des résultats obtenus sans occulter pour autant les obstacles rencontrés ou les problèmes qui restent à résoudre, sur le site lorrain notamment.

Notre communication ne vise que la campagne lancée au sein du réseau des archives publiques; elle n'évoquera donc pas directement le rôle joué par d'autres partenaires ainsi que par différentes structures associatives et patrimoniales, à l'exception du Centre minier de Lewarde, devenu un partenaire incontournable de la mémoire de la mine et qui conserve une partie non négligeable des archives minières du nord de la France.

\section{Une préoccupation ancienne}

L'intérêt manifesté par le réseau des archives, Archives nationales comme archives départementales, à l'égard des archives des houillères ne date évidemment pas de l'annonce de la disparition de Charbonnages de France. Depuis longtemps, il s'est préoccupé de leur conservation. Dans les années 1960, Bertrand Gille, qui dirige la section des archives d'entreprises des Archives nationales de 1950 à 1958, se penche sur le dossier et s'efforce de localiser les fonds des houillères. Cet effort de recensement sera poursuivi par son successeur à la tête du service, Isabelle Guérin-Brot '. C'est ainsi que les archives de certaines compagnies minières antérieures à la nationalisation sont remises aux Archives nationales et intégrées dans la série des archives d'entreprises avant d'être ultérieurement transférées au Centre des archives du monde du travail, à Roubaix. Parallèlement, d'autres fonds entrent, dès les années 1970, dans les séries J des archives départementales. Des contacts sont novés, des versements, encore partiels et limités, sont effectués. Bien évidemment, l'accent est mis sur la conservation des archives les plus anciennes, les documents du XVIIIle siècle et ceux du siècle suivant mais à cette époque, l'intervention des responsables des archives départementales doit se borner, pour l'essentiel, à des conseils en matière de traitement et d'éliminations. Ils manquent tout à la fois des moyens, des espaces et du personnel nécessaire pour accueillir l'ensemble des archives des houillères et mener dans ce domaine une politique dynamique.

1 Isabelle Guérin-Brot, «Les archives de l'industrie houillère ॥, 1978, 48 p., dactyl.
Parallèlement, au-delà des interventions ponctuelles des responsables des archives départementales légitimement soucieux d'assurer la sauvegarde des archives des unités d'exploitations situées dans leur département, la Direction des archives de France, au tournant des années 1990, commence à se préoccuper du sort des archives des houillères dans le but de donner une réponse globale et cohérente au problème posé par leur collecte et leur conservation. II ne s'agit plus en effet de prendre seulement en charge les archives historiques des compagnies minières d'avant guerre mais désormais d'éviter la déperdition d'un ensemble documentaire de premier ordre, encore cohérent, mais fragile, menacé, convoité et susceptible d'être dispersé.

Certes, dans le domaine des archives des entreprises publiques, le bilan de l'action de la Direction des archives de France apparaît quelque peu contrasté. Si elle est en effet consciente de l'intérêt présenté par ces archives pour la recherche, elle ne dispose pas des moyens suffisants, en locaux ou en personnel, pour traiter les masses particulièrement importantes de documents qu'elles représentent. Le projet de construction de cinq dépôts destinés aux archives d'entreprises, adopté en 1983 par le gouvernement, avait pu, un moment, laisser espérer que les archives des entreprises publiques pourraient être prises en charge mais l'abandon du projet après l'achèvement, en 1993, du seul Centre des archives du monde du travail de Roubaix, sonne le glas de cette politique ambitieuse. En dépit des dispositions législatives, il apparaît clairement que l'État ne possède pas les moyens de contrôler et de collecter ces archives. La Direction des archives de France doit donc se contenter d'actions de sensibilisation et d'incitations. Faute de véritables moyens d'action, elle borne son action à l'adoption de mesures limitées, se repose sur les initiatives de structures associatives, encourage la création de services d'archives au sein des entreprises. Dans le meilleur des cas, la négociation de conventions d'autonomie, plus ou moins formalisées, avec certaines entreprises publiques, les Houillères du Bassin du Nord et du Pasde-Calais en bénéficieront, maintient la fiction d'un suivi qui, dans les faits, faute d'actions de contrôle et de soutien, reste bien souvent virtuel.

Parmi les raisons qui favorisent pourtant l'intervention directe de la Direction des archives de France dans le dossier des archives des houillères, jove évidemment en premier lieu le statut même de ces archives. Depuis le 17 mai 1946, les compagnies minières ont en effet été nationalisées et les archives qu'elles 


\section{La sauvegarde de la mémoire de charbonnages de France}

produisent sont par conséquent des archives publiques, les archives des entreprises publiques ayant été intégrées dans le champ des archives publiques par la loi du 3 janvier 1979 désormais codifiée au livre II du Code du patrimoine ${ }^{2}$. Il appartient dans ces conditions à l'administration des archives d'exercer les missions fondamentales de contrôle et de collecte qui sont les siennes, de veiller à la bonne conservation de ces documents, de s'assurer de leur versement dans les services publics d'archives et de valider les éliminations proposées afin d'éviter soit des destructions intempestives, soit des appropriations indues.

Autre facteur d'intervention de la direction, le volume considérable des archives de Charbonnages de France qui exige certes une concertation globale avec les producteurs mais également avec les services d'archives concernés pour mettre en place au niveau national une politique cohérente en termes de collecte, de tris et d'éliminations.

La Direction des archives de France n'a pas eu besoin de mobiliser son réseau sensibilisé depuis longtemps à la question de la sauvegarde des archives des houillères mais elle était sensible aux inquiétudes exprimées par certains directeurs d'archives départementales du Bassin Centre-Midi sur les conséquences des mesures adoptées, de façon unilatérale, par les responsables des sites miniers. Ces inquiétudes portaient alors sur les conditions de transfert et de regroupement de certaines catégories d'archives à Saint-Étienne ainsi que sur les conditions des éliminations effectuées sans toujours le visa réglementaire au moment de la fermeture des sites.

Afin de répondre à ces interrogations mais aussi d'associer l'ensemble des services concernés à la démarche de sauvegarde entreprise, la Direction des archives de France a organisé, à partir de 1993 et de façon régulière, des réunions d'information avec les responsables des archives départementales. Ces réunions étaient destinées, ainsi que le précisait Philippe Bélaval à l'occasion de l'ouverture de la réunion du 6 décembre 1999, à « faire le point sur la situation des archives des houillères dans les différents bassins afin de déterminer la conduite de la Direction des archives de France à l'égard de Charbonnages de France et des houillères de bassin tant au niveau central que local ${ }^{3}$. En cela, la Direction assurait le rôle de coordination et d'impulsion qui est le sien.

2 Loi n 46-1072 du 17 mai 1946 portant nationalisation des exploitations de combustibles minéraux solides.

3 L'une des réunions d'information sera décentralisée à Saint-Étienne en 2004.
Troisième motif d'intervention dans le dossier, le risque fréquemment exposé par les archivistes départementaux, non sans fondements, d'un éclatement des fonds et d'une dispersion de documents à forte valeur symbolique et emblématique tels les plans et documents iconographiques. Comme pour les chantiers navals, la fermeture des houillères a provoqué des frustrations, des traumatismes qui comportent pour les archives des risques d'appropriation et de dispersion. Parallèlement, s'exprime fortement la volonté des élus et des populations de conserver ce patrimoine capable de restituer les traces d'un passé désormais révolu. Mais les efforts des particuliers ou des associations pour sauvegarder le patrimoine écrit des houillères risquent de contribuer paradoxalement à l'éclatement des fonds et à l'émiettement d'une information au détriment, à terme, de la recherche historique.

Enfin, et surtout, avec Charbonnages de France, la Direction des archives de France se trouve confrontée à la disparition programmée d'une entreprise publique qui a joué un rôle majeur dans l'économie du pays et dont il importe de sauvegarder la mémoire. Toutes ces raisons accumulées expliquent que la Direction des archives de France ait souhaité élaborer avec les responsables de l'entreprise un programme d'action véritablement concerté et cohérent. Ce programme doit mettre en œuvre les mesures susceptibles d'assurer la sauvegarde des archives d'une entreprise phare du secteur public et d'éviter la dispersion de documents essentiels à la compréhension de l'histoire économique et sociale de notre pays au cours des soixante dernières années. Reprenant les observations et suggestions faites par les responsables des archives départementales, la Direction propose ainsi à ses partenaires d'accompagner les dernières fermetures de sites d'un traitement véritablement archivistique des archives des houillères, basé notamment sur le respect des fonds et en étroite concertation avec le réseau des archives.

Or les préoccupations des archivistes rencontrent désormais celles des responsables de Charbonnages de France attentifs à la conservation de cette mémoire et soucieux d'en éviter la disparition. Faute de partenaires officiels bien identifiés et reconnus, les responsables des hovillères ont certes, longtemps, encouragé quand ils ne les ont pas suscités, les efforts de structures associatives militant pour la conservation de la mémoire de la mine. Dans le nord, ce sont les Houillères du bassin du Nord Pas-de-Calais qui provoquent ainsi la création du Centre historique minier 
de Lewarde conçu comme un conservatoire de la mine et de sa mémoire écrite. Mais une fois établis les contacts avec le réseau des archives, aussi bien à l'échelon national qu'au niveau départemental, les responsables de l'entreprise ont pris conscience de leurs responsabilités dans la conservation de cette mémoire. Ils ont été soucieux d'en assurer la transmission aux services d'archives appelés par la loi à les recevoir et disposant des moyens d'en assurer la conservation, la communication et la mise en valeur dans de bonnes conditions. De fait, les responsables de Charbonnages de France ont pris l'engagement d'apporter à la conservation des archives le même soin manifesté par eux dans le domaine de la mise en sécurité des installations minières ou de l'aménagement des friches industrielles.

Enfin, si la Direction souhaite, comme Charbonnages de France, répondre aux demandes exprimées par la population et les élus des bassins houillers afin de conserver une mémoire parfois douloureuse, mais toujours considérée comme profondément identitaire, elle se montre soucieuse, au-delà de sa bonne conservation, d'assurer une mise en valeur de cette mémoire par les historiens et les chercheurs certes mais également par le grand public.

La création, en 2004, d'un Etablissement public à caractère industriel et commercial (EPIC) unique Charbonnages de France, chargé de mettre en œuvre la liquidation de l'entreprise, a favorisé encore la coopération engagée avec le réseau des archives, ce réseau disposant désormais d'un interlocuteur unique en la personne du secrétaire général de l'entreprise.

\section{Un état des lieux}

La réalisation d'un état des lieux constituait le premier objectif à atteindre. Engagé dès 1999, cet état des lieux a été poursuivi et intensifié au cours des années suivantes, notamment après la réorganisation de la Direction des archives de France, par l'Inspection générale et le département de la politique archivistique et de la coordination interministérielle. II s'est traduit par une série de visites dans les différents lieux de stockage des archives de Charbonnages de France qui ont permis d'établir un diagnostic précis, d'évaluer l'importance des stocks d'archives concernées et de mettre en œuvre, en partenariat étroit avec l'entreprise, les mesures appropriées à la saine conservation de ses archives définitives.

La synthèse dressée à l'issue de cette enquête livre un paysage contrasté et souligne la diversité des solutions apportées, selon les lieux et les hommes, à la sauvegarde des archives des houillères. Cette diversité tient évidemment à l'histoire des bassins et notamment aux modalités de la fermeture des différents puits mais aussi à l'intervention, plus ou moins marquée, des collectivités territoriales et des services d'archives concernés. Elle met en lumière également sinon les incohérences, du moins les hésitations des politiques publiques dans le domaine.

\section{Bassin du Nord Pas-de-Calais}

C'est sans doute dans le bassin du Nord Pas-de-Calais que cette absence de cohérence apparaît de façon plus visible avec la création successive de deux structures voisines qui se partagent désormais la conservation des archives des houillères. En effet, c'est dans le milieu des années 1970 qu'un centre de la mémoire minière pour le Nord est envisagé par les responsables des houillères. Une structure associative, le Centre historique minier de Lewarde, voit ainsi le jour en 1982 sur le carreau de l'ancienne fosse Delhoye avec le soutien des houillères et des collectivités territoriales ${ }^{4}$. Le musée, devenu de nos jours le plus important musée de la mine en France, ouvre ses portes en mai 1984 avec initialement, sans doute, le simple projet de compléter les collections muséographiques en cours de constitution par une sélection de documents ${ }^{5}$. Rapidement toutefois, en liaison avec les fermetures successives des sièges et des puits, il prend en charge une grande quantité d'archives que lui remettent les houillères, archives administratives et techniques, plans, riches collections iconographiques du service communication, films...6.

Sans doute, la création du centre minier de Lewarde permettait-elle aux hovillères de disposer d'une structure assurant la conservation d'archives historiques dont le sort commençait à poser problème à I'heure où fermaient les puits et où les archives départementales du Nord et du Pas-de-Calais ne pouvaient les accueillir faute de place même si ces dernières avaient déjà pris en charge des versements parfois

4 Le centre a bénéficié notamment de financements de la part du ministère de la culture et de la communication (direction des musées de France), du conseil régional, du conseil général du Nord et enfin des Houillères du Nord Pasde-Calais, jusqu'à la disparition de ces dernières, à la fin de l'année 1992.

5 Direction des archives de France, dossier Mines; note de Georges Mouradian, responsable du Centre des archives du monde du travail de Roubaix, au directeur des archives de France, Roubaix, 21 décembre 1994.

6 La riche photothèque des houillères est remise au Centre historique minier en novembre 1992. 


\section{La sauvegarde de la mémoire de charbonnages de France}

conséquents. La Direction des archives de France donnait par ailleurs son aval à cette orientation puisque le 13 août 1985, elle accordait au directeur général des HBNPC (Hovillères du bassin du Nord et du Pas-de-Calais) une convention d'autonomie lui permettant de gérer lui-même ses archives, dans la mesure où, était-il précisé, les archives déposées au Centre historique minier de Lewarde sont gérées par une archiviste qualifiée sous le contrôle du directeur des archives départementales du Nord ${ }^{7}$.

Le Centre minier a en effet recruté une archiviste professionnelle qui, à la tête d'une petite équipe composée notamment de plusieurs agents mis à la disposition du centre par les houillères, organise un véritable service de préarchivage et fait déposer au centre minier les archives définitives ou les fonds destinés à être traités ultérieurement. Parallèlement, le Centre développe une active politique de collecte d'archives privées au même titre qu'il récolte les souvenirs personnels d'anciens mineurs et de leurs descendants ainsi que les archives de structures associatives.

Rapidement, le fonds d'archives ainsi constitué à Lewarde dont les éléments les plus anciens remontent à la seconde moitié du XVIII siècle, représente 2500 mètres linéaires auxquels il convient d'ajouter les fonds iconographiques, les films et les plans. La dévolution de ces archives publiques à une structure privée soulève cependant des interrogations au moment où les Houillères du Nord-Pas-de-Calais disparaissent en 1992. Le centre qui ne dispose bientôt plus d'archiviste professionnel, éprouve par ailleurs des difficultés à dominer la masse documentaire dont il est désormais responsable ${ }^{8}$.

Parallèlement, la Direction des archives de France avait lancé la construction du premier des cina centres dédiés aux archives du monde économique, campagne qui se bornera au seul Centre des archives du monde du travail de Roubaix. Disposant de capa-

7 Aux termes de l'article 10 du décret n 79-1037 du 3 décembre 1979 relatif à la compétence des services d'archives publics et à la coopération entre les administrations pour la collecte, la conservation et la communication des archives publiques, peuvent bénéficier en effet d'une convention d'autonomie leur permettant de gérer leurs archives, «les entreprises et établissements publics et les organismes de droit privé chargés de la gestion des services publics ou d'une mission de service public » avec l'accord de leur administration de tutelle et de la direction des archives de France.

8 Après le départ, en 1996, d'Annie Kuhnmunch, ancienne archiviste de Dunkerque, est évoquée, pendant plusieurs années, la nomination au CAMT de Roubaix d'un responsable scientifique susceptible d'être mis à la disposition du centre. cités de stockage et d'un personnel scientifique, le CAMT (Centre des archives du monde du travail) ne tarde pas à récupérer les archives minières confiées initialement aux archives départementales du Nord et du Pas-de-Calais avant de recevoir les archives des compagnies minières antérieures à la nationalisation conservées jusque là par la section des archives d'entreprises des Archives nationales dont celles des mines d'Anzin (1758-1949) ou celles des houillères et fonderies d'Avignon ou encore les archives de Decazeville. Tout naturellement, le CAMT s'investit dans le secteur minier, s'associant notamment à la campagne engagée par la Maison des mineurs de Lens en direction des archives des syndicats miniers.

L'articulation indispensable entre les deux centres voisins conduit ainsi, en juillet 1994, l'ingénieur général des mines, Paul-Louis Bourrelier, à préconiser, dans son rapport sur la politique d'archivage des données des anciennes exploitations minières $d u$ bassin du Nord et du Pas-de-Calais, la constitution d'un réseau avec la conservation des archives techniques et des plans à Lewarde, devenu centre de culture scientifique et technique de la mine et de l'énergie, et celle des archives administratives, sociales et financières à Roubaix. Grâce aux crédits européens, le Centre, qui a recruté une nouvelle archiviste, procède, de son côté, à d'importants travaux de restructuration. Les archives seront transférées en 2001 dans des locaux aménagés d'une capacité totale de 3200 mètres linéaires et dotés d'une véritable salle de consultation d'une capacité de dix places. Les préconisations du rapport Bourrelier ne sont cependant pas mises en œuvre et les chercheurs ne peuvent que déplorer la répartition totalement aléatoire des dossiers des anciennes compagnies entre les deux structures.

Pour en terminer avec le Nord, notons enfin la présence, à Noyelles-sous-Lens, des archives de l'ancienne Association nationale de gestion des retraités qui regroupe les dossiers du personnel des hovillères du Nord et du Pas-de-Calais ainsi que du Centre Midi, soit $21 \mathrm{~km}$. linéaires d'archives. L'association a été transformée, par la loi du 3 février 2004, en établissement public administratif, l'Agence nationale de garantie pour les droits des mineurs, devenue opérationnelle à

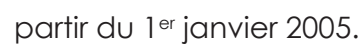

\section{Bassin de Lorraine}

Une autre solution a été retenue pour les houillères du bassin de Lorraine où progressivement, a été mise en place, à l'initiative des responsables des hovillères, un véritable service d'archives centralisé qui assure no- 
tamment la gestion des archives des compagnies antérieures à la nationalisation de 1946 avec des fonds prestigieux qui remontent au début du XIXe siècle.

À partir de 1985, comme dans le Nord, une structure associative, le centre de culture scientifique, technique et industrielle du bassin houiller de Lorraine, soutenu aussi bien par les houillères que par la DRAC Lorraine, se penche sur le problème de la conservation des archives des hovillères alors regroupées à Stiring, dans une ancienne caserne de uhlans. Elles le sont dans d'assez piètres conditions puisque la directrice des archives départementales indique à cette époque qu'elles sont « très négligées et en cours de dilapidation $\|^{9}$. Toutefois, à la différence du Nord, le statut public des archives des houillères est immédiatement rappelé et les offres présentées par les structures associatives pour se charger des archives, éludées. En définitive, les archives sont transférées au cours des années suivantes, en 1989-1990, dans de nouveaux locaux équipés à Freyming Merlebach après une campagne de tri qui a entraîné l'élimination de 4430 mètres linéaires d'archives ${ }^{10}$.

Lorsque s'achève la construction du CAMT de Roubaix, en 1993, les élus lorrains conservent un moment l'espoir de voir la région désignée pour accueillir un second centre interrégional d'archives du monde du travail susceptible de recevoir à la fois les archives des houillères et celles des industries sidérurgiques. La directrice des archives départementales de Moselle est alors chargée par la DRAC de faire une étude de faisabilité sur l'implantation d'un tel centre, au puits Simon à Forbach "'. En 1995, à une époque où, dans le cadre du projet d'entreprise, un groupe de travail est constitué au sein des houillères du bassin de Lorraine pour réfléchir à l'évolution du service des archives, les responsables des houillères sollicitent en vain de la Direction des archives de France la signature d'une convention d'autonomie. Résultat ou non de ces réflexions, en 1998, les hovillères lancent un appel d'offres pour la cession des activités de gestion des archives et de la documentation. L'appel d'offres s'étant révélé infructueux, les houillères transfèrent le

9 Direction des archives de France, dossier Mines ; lettre de la directrice des archives départementales de la Moselle, Lucie Roux, au directeur général des archives de France, Saint-Julien, 22 juin 1987.

10 Ibid., rapport du service archives documentation des houillères du bassin de Lorraine, 11 mars 1991 sur le bilan de l'opération de transfert des archives du bâtiment de Stiring dans les nouveaux locaux de Freyming Merlebach.

11 Le financement d'une étude de faisabilité du centre figure au contrat de plan État région signé le 11 juillet 1984. service des archives, en 1999, dans le magasin Jeanne d'Arc de Saint-Avold d'une capacité de $3600 \mathrm{~m}^{2}$. Au cours des mois qui suivent, sont ainsi regroupés sur le site 9000 mètres linéaires d'archives et 450000 plans. Ils seront rejoints, en 2002, par le très riche fonds audiovisuel pour lequel une salle climatisée est aménagée. Avec une équipe solide, le service d'archives de Saint-Avold qui dispose par ailleurs d'une petite salle de lecture, fait référence. Il est vrai que les directrices successives des archives départementales de la Moselle n'ont cessé d'assurer un contrôle scientifique qui a permis l'adoption et la généralisation des normes archivistiques. Saint-Avold accueille même 2500 m. I. d'archives privées, le site offrant en effet des prestations de stockage pour les archives des entreprises locales. Parmi ces fonds privés, figurent au demeurant les archives de plusieurs filiales des houillères dont celles de la cokerie de Carling.

\section{Le Centre-Midi}

Pour le bassin Centre Midi où ont été regroupées en 1969, au sein d'un établissement unique, les Houillères du Bassin Centre-Midi - les hovillères de bassin créées par la loi de nationalisation de 1946 dans le centre et le midi de la France - prévaut encore une autre organisation. Les HBCM ne disposent pas en effet de centre susceptible de regrouper toutes les archives de ses différents sites comme Roubaix et Lewarde pour le bassin du Nord-Pas-de-Calais ou Saint-Avold pour le bassin de Lorraine ${ }^{12}$. Compte tenu de l'indépendance géographique des exploitations, le versement des archives définitives des houillères dans les services publics d'archives est privilégié, du moins dans ceux qui disposent de suffisamment de place. Ainsi, les archives départementales de la Loire accueillent dès 1973-1974 plusieurs versements des hovillères de la Loire puis les archives du siège des HBCM établi à Saint-Étienne.

Dans les autres départements du Centre-Midi, la situation varie sensiblement avec des versements dans les différentes archives départementales mais également, faute de place dans ces dernières, des stockages partiels dans des bâtiments provisoires dans le Gard, à la Grand'Combe et la Saône-et-Loire, à Montceau-les-Mines. Ces annexes provisoires sont appelées à disparaître avec l'extension des bâtiments

12 II s'agissait des houillères des bassins d'Aquitaine, d'Auvergne, de Blanzy, des Cévennes, de Dauphiné, de la Loire et de Provence. Les dispositions du décret du 16 avril 1968 devinrent effectives au $1^{\text {er }}$ janvier 1969. 


\section{La sauvegarde de la mémoire de charbonnages de France}

des archives départementales concernées ${ }^{13}$. Dans le Tarn, à Carmaux, les archives sont regroupées dans une structure culturelle installée dans une ancienne clinique des houillères, sous le contrôle scientifique des archives départementales. En revanche, pour l'Hérault et l'Isère, les archives ont été regroupées pour des raisons de sécurité à Saint-Étienne en dépit des propositions faites par les archivistes départementaux pour les accueillir ${ }^{14}$.

Si la réalisation pour les HBCM d'un dépôt centralisé des archives est un moment envisagé, en 1993, sur le modèle de celui de la Lorraine, les responsables des houillères se contentent, en définitive, d'aménager à Saint-Étienne des locaux destinés à accueillir, à côté des archives du siège, les documents techniques en provenance des différents sites, les dossiers administratifs étant destinés pour leur part aux archives départementales.

Comme dans les autres bassins, l'intervention de structures muséographiques ou associatives qui militent pour la sauvegarde de la mémoire minière font, parfois, obstacle au versement des archives dans les services publics. C'est notamment le cas dans la Nièvre avec les archives de La Machine, dans l'Aveyron avec le musée de Decazeville et même à Saint-Étienne où le responsable des archives départementales doit réclamer au musée de la ville 150 mètres linéaires de plans récupérés par ce dernier.

À partir de 1998, avec les conseils d'une société prestataire de services qui met à la disposition de l'entreprise des archivistes diplômés, Charbonnages de France engage une campagne d'audits systématiques. Des correspondants archives sont désignés dans toutes les composantes de l'entreprise et reçoivent une formation appropriée ; les bordereaux de versement et d'élimination sont généralisés, le récolement des fonds est entrepris. Les procédures sont ainsi unifiées dans les différents sites.

Enfin, il convient de ne pas négliger, à côté des houillères de bassin, les archives centrales de Charbonnages de France transférées de Paris à Rueil-

13 Au moment où sont imprimés les actes de la présente journée d'étude, on doit déplorer la destruction presque complète, dans un incendie, des archives de l'annexe de Montceau-les-Mines.

14 Dans l'Isère, en 1993, une pétition est adressée au directeur général des archives de France par plusieurs maires du département inquiets du sort des archives historiques de l'unité d'exploitation Dauphiné, inquiétudes fondées si l'on en juge par le faible métrage linéaire des archives minières conservées en définitive pour ce département.
Malmaison en 1986 et qui représentent un ensemble particulièrement riche et volumineux.

On le voit, le tour d'horizon met en lumière des solutions divergentes apportées à la conservation des archives des houillères, un effort de concentration dans le Nord-Pas-de-Calais et surtout en Lorraine et une répartition territoriale dans le Centre Midi. L'étude met en relief également la gestion longtemps autonome de ses archives par les houillères sans véritable partenariat avec les services publics d'archives en matière de visas d'élimination notamment, ainsi que la prise en charge par différentes structures associatives d'archives publiques en contradiction avec les textes réglementaires.

\section{Un partenariat fructueux et dynamique}

Parallèlement à l'établissement d'un état des lieux, Charbonnages de France et la Direction des archives de France, désormais associés dans un partenariat actif, définissent un programme concerté qui repose sur les bases suivantes : la poursuite des travaux de tri et le versement dans les services d'archives publics des archives administratives dont la conservation définitive s'impose, le rétablissement des procédures des visas d'élimination, et enfin l'étude du problème posé par le sort des archives techniques indispensables à la gestion de l'après mine regroupées sur les sites de Billy-Montigny, Saint-Avold et Saint-Étienne.

Encadrées par la Société Alti, les équipes de Charbonnages de France entreprennent alors un travail considérable de tri et d'inventaire accompagné de l'établissement de bordereaux de versement et d'élimination soumis aux directeurs des services d'archives concernés. Cette campagne a été ainsi poursuivie et est en cours d'achèvement pour le Nord-Pasde-Calais et le Centre Midi.

Dans le nord, les archives administratives définitives regroupées sur le site de Billy-Montigny sont ainsi en cours de versement au CAMT. Les dossiers déjà versés au Centre historique minier de Lewarde y demeureront sous réserve d'ajustements destinés à regrouper les dossiers actuellement éclatés entre le Centre et le CAMT et du maintien des conditions actuelles de conservation matérielle et de traitement des archives. Une convention devra être passée avec ce dernier afin de régulariser la situation des archives publiques qu'il détient. II sera rappelé à cette occasion que les archives publiques de Charbonnages de France comprennent tous les documents confiés par les houillères du Nord Pas-de-Calais au centre minier, y compris les 
fonds iconographiques qui ne sauraient être dissociés des archives papier.

Dans le Nord comme dans le Centre-Midi, les services d'archives n'ont pris en charge que les archives définitives de l'entreprise. Il a été en effet décidé que Charbonnages de France conserverait jusqu'à sa disparition les archives courantes et intermédiaires dont la durée d'utilité administrative n'est pas échue. Les archives du siège de Charbonnages de France établi à Rueil-Malmaison après avoir longtemps été fixé à Paris, ont commencé à faire l'objet, pour leur part, de versements réguliers au CAMT naturellement désigné pour les recevoir ${ }^{15}$.

De la même façon, pour le Centre Midi, a été engagé sur une vaste échelle le versement aux archives départementales des archives administratives et techniques non utiles. Les chiffres suivants, non définitifs, permettent de mesurer l'importance des versements déjà réalisés ou prévus d'ici à 2007.

Bassin du Centre-Midi (chiffres communiqués par Charbonnages de France)

\begin{tabular}{|c|c|c|c|}
\hline & $\begin{array}{l}\text { versements } \\
\text { déjà effectués } \\
\text { (en mètres } \\
\text { linéaires) }\end{array}$ & $\begin{array}{l}\text { éliminations } \\
\text { réalisées } \\
\text { depuis } 1977\end{array}$ & $\begin{array}{l}\text { versements } \\
\text { à réaliser }\end{array}$ \\
\hline Allier & 0 & 10 & 14 \\
\hline Aveyron & 207 & 140 & 20 \\
\hline Bouches-du-Rhône & 217 & 284 & 58 \\
\hline Gard & 165 & 181 & 746 \\
\hline Hérault & 18 & 0 & 13 \\
\hline Isère & 53 & 6 & 22 \\
\hline Loire & 1117 & 244 & 612 \\
\hline Nièvre & 350 & 0 & 0 \\
\hline Puy-de-Dôme & 27 & 0 & 0 \\
\hline Saône-et-Loire & 584 & 593 & 0 \\
\hline Tarn & 1676 & 83 & 0 \\
\hline Total & 4414 & 1541 & 1485 \\
\hline
\end{tabular}

15 À la fin du mois d'avril 2006, 451 mètres linéaires avaient déjà été dirigés sur Roubaix et 626 mètres linéaires avaient été régulièrement détruits.
Pour la Lorraine, dans l'état actuel des choses, le bâtiment de Saint-Avold, géré désormais par Charbonnages de France, devrait être maintenu avec l'ensemble des documents qu'il contient, y compris donc les archives techniques qui s'y trouvent déjà ${ }^{16}$. Des négociations sont en cours pour mettre au point les modalités du transfert juridique de ces archives ; la commune de Saint-Avold souhaite en effet conserver sur son territoire ce très riche fonds d'archives et faire l'acquisition du bâtiment qui les abrite. Une solution devrait être trouvée avant 2007-2008 associant l'État, les différentes collectivités intéressées, commune, conseil général et région.

Enfin, les dossiers personnels des mineurs, conservés à Noyelles-sous-Lens par l'ANGDM (Agence nationale pour la garantie du droit des mineurs), feront l'objet de tris, voire d'échantillonnages dont les modalités seront déterminées par la Direction des archives de France sur la base des expérimentations en cours aussi bien au CAMT qu'en Moselle. On peut par ailleurs s'interroger sur la pertinence d'une conservation centralisée des dossiers des mineurs et sur l'opportunité d'un regroupement de ces dossiers avec ceux des compagnies minières auxquels ils se rapportent. Ce point reste à trancher. De la même façon, dans un souci de cohérence, il a été décidé, afin de reconstituer les fonds et de faciliter la recherche historique, de faire retour des archives des compagnies privées antérieures à la nationalisation et conservées au CAMT dans les départements qui possèdent les archives des houillères qui ont pris la suite de ces compagnies après la nationalisation de 1946.

\section{Les archives techniques des mines}

Un statut particulier a été réservé aux archives techniques des mines. La gestion de l'après mine exige en effet que soient rendus immédiatement accessibles les documents indispensables à la gestion de situations de crise provoquées par les affaissements miniers. En définitive, la prise en charge de ces archives par les archives départementales a été écartée en raison des obligations trop lourdes qu'elle entraînerait pour ces services qui ne disposent pas du personnel possédant les connaissances techniques nécessaires pour analyser et transmettre rapidement les informations contenues dans ces documents. Déjà, en 1993, le directeur des archives de France considérait qu'il appartenait à Charbonnages de France de gérer les

16 Les Houillères du Bassin de Lorraine ont été dissoutes à compter du 1er mars 2004 (loi du 3 février 2004 et arrêté du 24 février 2004). 


\section{La sauvegarde de la mémoire de charbonnages de France}

plans techniques des mines et d'exercer les responsabilités juridiques et techniques liées à leur détention ${ }^{17}$.

Il a été envisagé de confier la conservation de ces archives à l'Agence de prévention et de surveillance des risques miniers mais cette solution n'a pas été retenue par le ministère de l'Industrie ${ }^{18}$. En définitive, c'est au BRGM (Bureau de recherches géologiques et minières) dont les missions ont été récemment redéfinies, qu'il a paru préférable de confier les archives techniques ${ }^{19}$. Ce choix a été confirmé par le ministre lors d'un débat au Sénat sur l'après mine le 24 novembre 2004. Sur la proposition faite par le sénateur Philippe Leroy au cours de ce débat, un groupe de travail a été constitué au début de l'année 2005 et la responsabilité en a été confiée à Charbonnages de France. Les conclusions de ce groupe de travail reprennent les principales dispositions évoquées lors du débat au Sénat.

Le BRGM doit assurer la conservation des archives techniques qui lui sont confiées dans plusieurs centres répartis sur le territoire : Billy-Montigny pour le Nord et Pas-de-Calais, Orléans, son siège, pour les archives des puits du Grand Ouest, du Sud-Ouest et du Massif central, et Gardanne pour les sites du sud de la France. Pour la Lorraine, il est prévu de maintenir dans le

17 Direction des archives de France, dossier Mines ; lettre du directeur des archives de France à Jean-Pierre Kucheida, député du Pas-de-Calais et président de l'Association des communes minières de France. Dans le bassin du Nord-Pasde-Calais, un accord avait été conclu dans les années 1970 entre le BRGM et les HBNPC aux termes duquel les plans du sous-sol devaient être remis au BRGM et envoyés à son siège d'Orléans au fur et à mesure de la fermeture des puits.

18 L'agence était chargée en vertu de l'article 4 de la loi 99-245 du 30 mars 1999 de recueillir et de conserver les documents mentionnés à l'article 91 du code minier. Ce dernier organise la procédure d'arrêt des travaux miniers et prévoit les informations qui doivent constituer le dossier de déclaration d'arrêt des travaux. Le décret 2002-353 du 15 mars 2002 limite les attributions de l'agence aux documents cités par la procédure d'arrêt, à compter de la date de publication du décret 2001-209 du 6 mars 2001 modifiant le décret 95-696 du 9 mai 1995 relatif à l'ouverture des travaux miniers et à la police des mines. L'agence a été supprimée en 2007.

19 Décret 2004-991 du 20 septembre 2004 modifiant le décret 59-1205 du 23 octobre 1959 relatif à l'organisation administrative et financière du Bureau de recherches géologiques et minières (BRGM). L'article 3 précise que le BRGM est chargé de recueillir directement ou auprès d'autres détenteurs, valider, archiver et mettre à la disposition des usagers sous une forme appropriée les informations couvrant le territoire national ainsi que le plateau continental parmi lesquelles celles concernant les fouilles, forages et levers géologiques recueillis en application du code minier. bâtiment de Saint-Avold les archives techniques qui y sont déjà conservées. Les autres archives techniques qui n'ont pas déjà fait l'objet d'un versement et celles des Mines domaniales de potasse d'Alsace et des mines privées seront rassemblées pour leur part sur un autre site, à Vouters. II a été cependant convenu que les archives techniques « utiles » déjà conservées dans les fonds qui ont fait l'objet de versements et de classements dans les services d'archives publics, n'en seraient pas retirées et ne seraient pas remises au BRGM ; en revanche, elles pourront être reproduites à ses frais s'il le souhaite.

Afin de disposer très rapidement d'informations opérationnelles, la réalisation d'une base de données recensant tous les documents techniques disponibles sur les mines au sens large du terme a été prévue. Cette base doit préciser pour chacun des documents recensés leur origine exacte ainsi que les cotes des dossiers dont ils ont été extraits. Cette base à laquelle il est prévu que les services d'archives départementales concernés soient reliés, pourra, à terme, prendre en compte les références des documents techniques conservés dans ces services ainsi qu'au Centre historique minier de Lewarde.

Les « archives techniques dites utiles » confiées au BRGM sont essentiellement constituées de plans mais elles comprennent également des plans cadastraux, des journaux de mines, des registres d'exploitation, des dossiers de dégâts miniers. Elles recouvrent également des documents anciens, ceux qui correspondent à l'exploitation des couches les moins profondes du sous-sol, et qui, précisément, sont susceptibles des désordres les plus importants. II convient de préciser par ailleurs que tous les plans des houillères n'entrent pas dans la catégorie des archives techniques « utiles »: ceux des bâtiments vendus ou aliénés, des chevalets, usines et installations techniques détruites ou démontées sont versés dans les services d'archives.

Une semblable scission des fonds, constitue cependant une atteinte à l'un des principes de base de la pratique archivistique dans notre pays, celui du respect des fonds. La Direction des archives de France a souhaité, dans ces conditions, conditionner son accord à la mise en œuvre d'un système de classement des archives techniques par le BRGM qui puisse assurer la traçabilité pérenne des documents. La nécessité d'identifier parfaitement les documents techniques confiés au BRGM répond par ailleurs au souci de la direction d'en permettre la réintégration ultérieure dans les fonds dont ils relevaient initialement, désormais conservés par les services publics d'archives. 
Si les solutions retenues allient les contraintes de la gestion de l'après mine aux règles de conservation des archives publiques, différents problèmes n'ont pas encore trouvé de solution : le sort archivistique des dossiers médicaux des mineurs actuellement conservés à l'AHNAC (Association hospitalière du Nord-Artois Cliniques) et entreposées à Flers-en-Escrebieux ou celui des archives anciennes des filiales des hovillères. La Direction des archives de France souhaite en effet que des conventions soient conclues avec les sociétés désormais propriétaires de ces filiales, toujours dans le but que les documents de conservation définitive antérieurs à la privatisation puissent, à terme, rejoindre les services publics d'archives.

Dernier domaine dans lequel le réseau des archives doit se montrer particulièrement vigilant, les fonds iconographiques constitués de plaques de verre, de clichés photographiques, de films, de réalisations audiovisuelles, souvent très riches. II est tentant en effet pour les collectivités et pour les associations d'en demander la rétrocession. Il convient, dans ces conditions, de rappeler que ces documents font partie intégrante des fonds d'archives, qu'ils ont un caractère public et qu'ils ne peuvent être aliénés sous quelque forme que ce soit. Les possibilités techniques actuelles permettent au demeurant de réaliser toutes les reproductions souhaitables et de répondre aux demandes des élus et des structures associatives.

Il est évidemment trop tôt pour dresser un bilan de l'opération en cours. Bien des questions restent encore en suspens et des solutions devront être trouvées d'ici la disparition de Charbonnages de France : le sort du bâtiment de Saint-Avold, celui des filiales de l'entreprise, la réalisation effective de la base de données... ${ }^{20}$. II n'en demeure pas moins que grâce à une approche pragmatique adoptée par les différents partenaires, grâce à la coopération intelligente développée entre l'entreprise et le réseau des archives, l'essentiel des archives des houillères a déjà rejoint ou est en train de rejoindre les collections publiques. Une part essentielle de la mémoire économique du pays aura ainsi été sauvegardée.

Il convient de salver le formidable effort consenti par les responsables des services d'archives territoriaux comme par le Centre du monde du travail de Roubaix pour prendre en charge les fonds volumineux des houillères et l'investissement non moins important que représentera le traitement de ces archives sans oublier la part prise dans cette action de sauvegarde par le réseau associatif. II appartiendra ensuite aux chercheurs et aux structures culturelles, de mettre en valeur cette mémoire accumulée et de faire connaître aux jeunes générations et au grand public l'histoire de la mine. En conclusion, un vœu pourrait être formulé : la réalisation d'un guide des sources de l'histoire de la mine que la richesse du patrimoine écrit minier rend éminemment souhaitable.

20 Depuis la journée d'étude sur les archives des mines, le bâtiment de Saint-Avold est devenu une annexe des archives départementales de la Moselle. Il doit abriter également les archives de la commune de Saint-Avold. 